\title{
On exponential sums with multiplicative coefficients, II
}

\author{
by \\ Gennady Bachman (Las Vegas, NV)
}

0. Introduction. Let $\mathcal{F}$ be the class of complex-valued multiplicative functions $f$ satisfying $|f| \leq 1$. For each $f \in \mathcal{F}$ and for any real numbers $x \geq 3$ and $\alpha$ we let $F(x, \alpha)$ denote the exponential sum

$$
F(x, \alpha)=\sum_{n \leq x} f(n) e(\alpha n),
$$

where $e(t)$ stands for $e^{2 \pi i t}$. New bounds for $F(x, \alpha)$ have been announced by the author in [Ba1] and the purpose of this paper is to supply proofs for these estimates.

The problem of obtaining bounds for $F(x, \alpha)$ uniform in $f \in \mathcal{F}$ has been first considered by H. Daboussi. He showed [Da1] (see also [DD1] and [DD2]) that if $|\alpha-s / r| \leq 1 / r^{2}$ and $3 \leq r \leq(x / \log x)^{1 / 2}$, for some coprime integers $s$ and $r$, then, uniformly for all $f \in \mathcal{F}$, we have

$$
F(x, \alpha) \ll \frac{x}{\sqrt{\log _{2} r}},
$$

where we write $\log _{k}, k=2,3$, for the $k$ th iterate of the logarithmic function. Although far from optimal (see below), this estimate immediately yields the following very interesting corollary. For every irrational $\alpha$ we have

$$
\lim _{x \rightarrow \infty} \frac{1}{x} F(x, \alpha)=0
$$

uniformly for all $f \in \mathcal{F}$.

It was later observed by $\mathrm{G}$. Tenenbaum $[\mathrm{Te}]$ that this result provides some measure of independence of the additive and multiplicative structures of the set of integers. More precisely, he formulated the following question.

2000 Mathematics Subject Classification: 11L07, 11N99.

I would like to thank Professors Andrew Granville and Gérald Tenenbaum for helpful discussions about various topics related to this project. I especially wish to thank Professor Adolf Hildebrand for suggesting this problem in the first place, and for numerous discussions on this and related topics over the course of this project. 
Writing

$$
\frac{1}{x} F(x, \alpha)=\left(\frac{1}{x} \sum_{n \leq x} f(n)\right)\left(\frac{1}{x} \sum_{n \leq x} e(\alpha n)\right)+o(1),
$$

we ask what can be said about the error term. In particular, we would like to characterize those functions $f$ such that for every irrational $\alpha$ we have

$$
\frac{1}{x} F(x, \alpha)=o\left(\frac{1}{x}\left|\sum_{n \leq x} f(n)\right|\right) .
$$

(Observe that (0.2) implies (0.3) only for those functions $f$ for which $\sum_{n \leq x} f(n) \asymp x$.) The question of when (0.3) holds was first raised in a paper of Y. Dupain, R. R. Hall and G. Tenenbaum [DHT]. It was shown there, among other things, that (0.3) holds for the special case of the function $f$ given by $n \mapsto y^{\Omega(n)}$, where $\Omega(n)$ denotes the total number of prime factors of $n$ and $0<y<2$ is fixed. Another interesting special case is when $f$ is a characteristic function of integers free of prime factors greater than $y \geq 2$. Sharp estimates for the corresponding exponential sum have been obtained by E. Fouvry and G. Tenenbaum [FT] providing a quantitative version of (0.3) for a wide range of parameters $x$ and $y$.

The problem of characterizing functions $f$ for which the asymptotic relation (0.3), or even the relation

$$
F(x, \alpha)=o\left(\sum_{n \leq x}|f(n)|\right)
$$

hold appears to be rather difficult, and the known results are rather restrictive. It was shown by Daboussi [Da2] that if $0<y<2$ is fixed, and if $f$ is a completely multiplicative function with $|f(p)|=y$ for all primes $p$, then for every irrational $\alpha$ the relation $(0.3)^{\prime}$ holds. This was improved by L. Goubin [Go] who showed that the same conclusion holds for any multiplicative function $f$ satisfying the three conditions: $|f(p)| \leq y, \sum_{p}(y-|f(p)|) / p<\infty$, and $\sum_{p} \sum_{\nu=2}^{\infty}\left|f\left(p^{\nu}\right)\right| p^{-\nu}\left(\log p^{\nu}\right)^{\max (1-y, 0)}<\infty$.

On the other hand, a spectacular advance was achieved by H. L. Montgomery and R. C. Vaughan [MV] who improved the original estimate of Daboussi (0.1) as follows. Suppose that $|\alpha-s / r| \leq 1 / r^{2}$ and $2 \leq R \leq r \leq$ $x / R$ for some coprime integers $s$ and $r$. Then we have

$$
F(x, \alpha) \ll \frac{x}{\log x}+\frac{x}{\sqrt{R}}(\log R)^{3 / 2},
$$

uniformly for all $f \in \mathcal{F}$. Furthermore, they showed that aside from the logarithmic factor this bound is sharp. More precisely, they established the following propositions: 
(i) For any real $x \geq 3$ and any $\alpha$ there is an $f \in \mathcal{F}$ such that $|F(x, \alpha)| \gg$ $x / \log x$.

(ii) If $r \leq \sqrt{x}$ and $(s, r)=1$, then there is an $f \in \mathcal{F}$ for which $|F(x, s / r)| \gg x / \sqrt{r}$.

(iii) If $x /(\log x)^{3} \leq T \leq x$, then there exist coprime integers $s$ and $r$ and $f \in \mathcal{F}$ such that $T-3 x / T \leq r \leq T$ and $|F(x, s / r)| \gg \sqrt{x T}$.

Motivated by (0.4) we can propose a quantitative form of $(0.3)^{\prime}$ as follows. Characterize those functions $f$ for which the inequality

$$
F(x, \alpha) \ll \frac{x}{\log x}+\frac{1}{\sqrt{R}} \sum_{n \leq x}|f(n)|
$$

holds, where $x, \alpha$ and $R$ satisfy the hypotheses of (0.4). Let us immediately note that $(0.5)$ certainly does not hold for all $f \in \mathcal{F}$. This follows readily by fixing a natural number $r$ and considering a completely multiplicative function $f$ whose values on primes is given by $f(p)=1$, if $p \equiv 1(\bmod r)$, and 0 , otherwise. Thus

$$
F\left(x, \frac{1}{r}\right)=e\left(\frac{1}{r}\right) \sum_{n \leq x} f(n)=e\left(\frac{1}{r}\right) \sum_{\substack{n \leq x \\ p \mid n \Rightarrow p \equiv 1(\bmod r)}} 1,
$$

and hence (0.5) with $R=r$ will hold only if the last summation over $n$ is $\ll x / \log x$. But, for $x$ sufficiently large, it is not difficult to see that the relation

$$
\sum_{\substack{n \leq x \\ p \mid n \Rightarrow p \equiv 1(\bmod r)}} 1 \asymp \frac{x}{\varphi(r)}(\log x)^{1 / \varphi(r)-1}
$$

holds, where $\varphi$ is Euler's totient function.

In view of this example it is evident that a necessary condition for the validity of $(0.5)$ is that $f$ is supported on a positive proportion of primes up to $x$, viz.

$$
\sum_{p \leq x} \frac{|f(p)|}{p} \geq \lambda \log _{2} x
$$

for some fixed $\lambda, 0<\lambda \leq 1$. We believe that this condition may also be sufficient and formulate the following conjecture. Let $\mathcal{F}_{\lambda}(x)$, for $x \geq 3$ and $0<\lambda \leq 1$, be the subclass of $\mathcal{F}$ consisting of functions $f$ satisfying (0.6).

ConjeCture. Let $x \geq 3, \alpha$ and $R \geq 3$ be real numbers and suppose that $|\alpha-s / r| \leq 1 / r^{2}$ and $R \leq r \leq x / R$ for some coprime integers $s$ and $r$. Then

$$
F(x, \alpha) \ll \lambda \frac{x}{\log x}+\frac{1}{\sqrt{R}} \sum_{n \leq x}|f(n)|,
$$

uniformly for all $f \in \mathcal{F}_{\lambda}(x)$. 
1. Statement of results. Given $f \in \mathcal{F}$ set

$$
S(x)=\sum_{p \leq x} \frac{|f(p)|}{p} \quad \text { and } \quad S_{q}(x)=\sum_{\substack{p \leq x \\(p, q)=1}} \frac{|f(p)|}{p} .
$$

By an estimate of R. R. Hall [Ha] we have

$$
\sum_{n \leq x}|f(n)| \ll \frac{x}{\log x} e^{S(x)} \asymp x \exp \left(-\sum_{p \leq x} \frac{1-|f(p)|}{p}\right),
$$

uniformly for all $f \in \mathcal{F}$. Furthermore, this bound is sharp in the sense that there are functions $f$ for which (1.1) holds with « replaced by $\gg$. Principal results of this paper (Corollaries 1 and 2) give a weaker form of the conjecture with (0.7) modified by substituting (1.1). We begin, however, with estimates valid for the entire class $\mathcal{F}$.

TheOREM 1. Let $x \geq 3, \alpha$ and $\varepsilon>0$ be real numbers and set $Q=$ $x /(\log x)^{3}$. Furthermore, let $a$ and $q$ be coprime integers satisfying $1 \leq q \leq Q$ and $|\alpha-a / q| \leq 1 /(q Q)$. Then

$$
F(x, \alpha) \ll_{\varepsilon} \frac{x}{\log x}+\frac{x}{\sqrt{q}(\log x)^{1-\varepsilon}}+\frac{x}{\sqrt{q} \log x} e^{S_{q}(x)}\left(\frac{q}{\varphi(q)}\right)^{3 / 2},
$$

uniformly for all $f \in \mathcal{F}$.

Our second estimate has a wider range of applicability at the expense of a somewhat weaker bound.

TheOREM 2. Let $x \geq 3, \alpha, R \geq 3$ and $\varepsilon>0$ be real numbers and suppose that $|\alpha-s / r| \leq 1 / r^{2}$ and $R \leq r \leq x / R$ for some coprime integers $s$ and $r$. Then

$$
F(x, \alpha) \ll_{\varepsilon} \frac{x}{\log x}+\frac{x}{\sqrt{R}(\log x)^{1-\varepsilon}}+\frac{x}{\sqrt{R} \log x} e^{S(x)}(\log R)^{1 / 2}\left(\log _{2} R\right)^{3 / 2},
$$

uniformly for all $f \in \mathcal{F}$.

Before continuing our main line of results we take a small detour and observe that if $f$ is not supported on a positive proportion of primes up to $x$, e.g., $S(x) \asymp \log _{3} x$, then a better estimate is given by the following:

TheOREM 3. Let $x \geq 3, \alpha$ and $R \geq 3$ be real numbers and suppose that $|\alpha-s / r| \leq 1 / r^{2}$ and $R \leq r \leq x / R$ for some coprime integers $s$ and $r$. Then

$$
F(x, \alpha) \ll \frac{x}{\log x}+\frac{x}{\sqrt{R} \log x} e^{S(x)}(\log R)^{1 / 2}\left(\log _{2} R\right)\left(\log _{2} x\right)^{1 / 2},
$$

uniformly for all $f \in \mathcal{F}$. 
Thus Theorem 3 gives a better estimate when $S(x)$ is "small" and by combining this theorem and Theorem 2 one obtains a bound superior in general to each of them individually.

We now return to the main focus of this paper, the classes $\mathcal{F}_{\lambda}(x)$. For $f \in \mathcal{F}_{\lambda}(x)$ Theorems 1 and 2 immediately yield the following corollaries, respectively:

Corollary 1. Let $x \geq 3$ and $\alpha$ be real numbers and set $Q=x /(\log x)^{3}$. Furthermore, let $a$ and $q$ be coprime integers satisfying $1 \leq q \leq Q$ and $|\alpha-a / q| \leq 1 /(q Q)$. Then

$$
F(x, \alpha) \ll_{\lambda} \frac{x}{\log x}+\frac{x}{\sqrt{q} \log x} e^{S_{q}(x)}\left(\frac{q}{\varphi(q)}\right)^{3 / 2},
$$

uniformly for all $f \in \mathcal{F}_{\lambda}(x)$.

Corollary 2. Let $x \geq 3, \alpha$ and $R \geq 3$ be real numbers and suppose that $|\alpha-s / r| \leq 1 / r^{2}$ and $R \leq r \leq x / R$ for some coprime integers $s$ and $r$. Then

$$
F(x, \alpha) \ll_{\lambda} \frac{x}{\log x}+\frac{x}{\sqrt{R} \log x} e^{S(x)}(\log R)^{1 / 2}\left(\log _{2} R\right)^{3 / 2},
$$

uniformly for all $f \in \mathcal{F}_{\lambda}(x)$.

Next we address the accuracy of these estimates. In view of the second inequality in (1.1) it is evident that Corollary 2 provides a stronger bound than (0.4) even in the case when $S(x)$ is maximal. In particular, this shows that our estimates are quite sharp, since we already noted that (0.4) was. Furthermore, given a fixed real number $\lambda, 0<\lambda \leq 1$, the original examples of Montgomery and Vaughan yielding propositions (i)-(iii) can be easily modified to establish the following versions, respectively:

(i) $)^{\prime}$ For any real $x \geq 3$ and any $\alpha$ there is an $f \in \mathcal{F}$ with $S(x)=$ $\lambda \log _{2} x+O(1)$ such that $|F(x, \alpha)| \gg x / \log x$.

(ii) $)^{\prime}$ If $q \leq \exp \left((\log x)^{\lambda / 3}\right)$ and $(a, q)=1$, then there is an $f \in \mathcal{F}$ with $S(x)=\lambda \log _{2} x+O(1)$ for which

$$
|F(x, a / q)| \gg_{\lambda} \frac{1}{\sqrt{q}} \sum_{\substack{n \leq x \\(n, q)=1}}|f(n)| \frac{q}{\varphi(q)} \gg_{\lambda} \frac{x}{\sqrt{q} \log x} e^{S_{q}(x)} \frac{q}{\varphi(q)} .
$$

(iii) $^{\prime}$ If $x /(\log x)^{3} \leq T \leq x$, then there exist coprime integers $s$ and $r$ and $f \in \mathcal{F}$ with $S(x)=\lambda \log _{2} x+O(1)$, such that $T-3 x / T \leq r \leq T$ and

$$
|F(x, s / r)| \gg_{\lambda} \frac{1}{\sqrt{x / T}} \sum_{n \leq x}|f(n)| \gg_{\lambda} \frac{\sqrt{x T}}{\log x} e^{S(x)} .
$$


We summarize these facts somewhat colloquially by saying that our estimates are sharp "throughout" the class of functions supported on a positive proportion of primes up to $x$ in the sense that (0.4) is sharp only for the subclass $\mathcal{F}_{1}(x)$.

On the other hand, our results imply neither $(0.7)$ nor $(0.3)^{\prime}$. Of course, we do get a weaker form of $(0.7)$ for those functions $f \in \mathcal{F}_{\lambda}(x)$ for which

$$
\sum_{n \leq x}|f(n)| \asymp \frac{x}{\log x} e^{S(x)} .
$$

Thus, for example, one readily sees using standard methods that functions $f$ for which Daboussi and Goubin established $(0.3)^{\prime}$ (with $0<y \leq 1$ ) do satisfy this condition. In general, however, no useful simple criterion which implies (1.2) is known, and determining such is itself an interesting problem. In particular, it is certainly not true that (1.2) holds for all $f \in \mathcal{F}_{\lambda}(x)$.

As we have already mentioned, Corollary 2 provides a slight improvement on (0.4) even in the case when $S(x)$ is maximal or, more generally, when $e^{S(x)} \asymp \log x$. For this important special case we can offer further improvements as follows:

THeOREM 4. Let $x \geq 3$ and $\alpha$ be real numbers and set $Q=x /(\log x)^{3}$. Furthermore, let $a$ and $q$ be coprime integers satisfying $1 \leq q \leq Q$ and $|\alpha-a / q| \leq 1 /(q Q)$. Then

$$
F(x, \alpha) \ll \frac{x}{\log x}+\frac{x}{\sqrt{\varphi(q)}},
$$

uniformly for all $f \in \mathcal{F}$.

TheOREm 5. Let $x \geq 3, \alpha$ and $R \geq 3$ be real numbers and suppose that $|\alpha-s / r| \leq 1 / r^{2}$ and $R \leq r \leq x / R$ for some coprime integers $s$ and $r$. Then

$$
F(x, \alpha) \ll \frac{x}{\log x}+\frac{x}{\sqrt{R}}\left(\log R \log _{2} R\right)^{1 / 2},
$$

uniformly for all $f \in \mathcal{F}$.

2. Preliminaries. Lemmas $1-3$ below are principal ingredients in the proofs of Theorems 1-5. The first lemma gives a bound for the exponential sum $F(x, \alpha)$ in terms of sums of non-negative multiplicative functions in short intervals and arithmetic progressions. This estimate improves on an earlier estimate of the author [Ba2, Lemma 1]. Our new argument is simply a strengthening of the argument given in [Ba2], and as such it relies fundamentally on the original estimate of Montgomery and Vaughan (0.4). This modification, however, is quite crucial and the new bound is essentially sharp. 
We first introduce some definitions. We will find it convenient to write, for $x \geq 3$,

$$
\mathcal{L}=\log x, \quad \mathcal{L}_{2}=\log _{2} x, \quad Q=x / \mathcal{L}^{3} .
$$

Fix a sufficiently large real number $x_{0}$ such that for all $x \geq x_{0}$ the inequality $Q \geq 1$ holds. Given real numbers $x \geq x_{0}$ and $\alpha$, by Dirichlet's theorem there exist integers $a$ and $q$ such that

$$
(a, q)=1, \quad 1 \leq q \leq Q, \quad|\alpha-a / q| \leq 1 /(q Q) .
$$

Set

$$
\beta=\alpha-a / q
$$

so that $|\beta| \leq 1 /(q Q)$, and define

$$
\bar{x}=\min (x, 1 /|\beta|) .
$$

Finally, for a real number $P$ we use the notation $p$ (respectively $l) \sim P$ to denote prime (respectively natural) numbers in the interval $(P, 2 P]$.

LEMMA 1. Let $x \geq x_{0}$ and $\alpha$ be real numbers and let $a$ and $q$ be integers satisfying (2.1). Then

$$
\begin{aligned}
& F(x, \alpha) \ll \frac{x}{\mathcal{L}}+\frac{1}{\mathcal{L}} \sqrt{\frac{q}{\varphi(q)}} \sum_{i: 1 \leq 2^{i}<x /(q x / \bar{x})^{2}} 2^{i} \\
& \times\left(\sum_{1 \leq j \leq\lceil x / \bar{x}\rceil} \frac{1}{j} \sum_{\substack{n, m \leq x / 2^{i} \\
(j-1) \bar{x} / 2^{i} \leq|n-m|<j \bar{x} / 2^{i} \\
n \equiv m(\bmod q)}}|f(n)| \cdot|f(m)|\right)^{1 / 2},
\end{aligned}
$$

uniformly for all $f \in \mathcal{F}$.

Proof. If $q \geq \mathcal{L}^{3}$, the conclusion of the lemma follows immediately from (0.4), applied with $s=a, r=q$ and $R=\mathcal{L}^{3}$. Thus it remains to consider the case when $q<\mathcal{L}^{3}$.

As we have indicated earlier, much of the proof of this result has been already carried out in [Ba2, Proof of Lemma 1]. In particular, it was shown there $[\mathrm{Ba} 2,(2.6),(2.7),(2.12)]$ that the estimate

$$
F(x, \alpha)=\frac{1}{\mathcal{L}} \sum_{i: x_{2} \leq 2^{i}<x_{3}} \sum_{p \sim 2^{i}} f(p) \log p F(x / p, \alpha p)+O(x / \mathcal{L})
$$

holds, where $x_{2}$ and $x_{3}$ are given by

$$
x_{2}=\exp \left\{(q x / \bar{x})^{1 / 5}\right\}, \quad x_{3}=x /(q x / \bar{x})^{2}
$$

(we use the same indices as in [Ba2] where $x_{1}$ was another parameter, [Ba2, $(2.7)])$. Applying Cauchy's inequality to the inner sum in (2.4) we obtain, 
as before,

$$
\begin{aligned}
\left|\sum_{p \sim 2^{i}} f(p) \log p F(x / p, \alpha p)\right|^{2} & \\
& \leq \sum_{p \sim 2^{i}}|f(p)|^{2} \log p \sum_{p \sim 2^{i}} \log p|F(x / p, \alpha p)|^{2} \\
& \ll 2^{i} \sum_{p \sim 2^{i}} \log p\left|\sum_{n \leq x / p} f(n) e(\alpha p n)\right|^{2} .
\end{aligned}
$$

At this point in [Ba2] we proceeded to estimate the summation on the right-hand side of (2.6) by squaring out the inner sum, swapping the order of summations and then estimating the new inner sum by standard methods. Our new idea is to introduce the weights $w(l)$ defined by

$$
w(l)= \begin{cases}\log l & \text { if } l \text { is prime, } \\ q / \varphi(q) & \text { if } l \text { is not prime and }(l, q)>1 \\ 0 & \text { otherwise, }\end{cases}
$$

and write

$$
\begin{aligned}
\sum_{p \sim 2^{i}} \log p \mid & \left.\sum_{n \leq x / p} f(n) e(\alpha p n)\right|^{2} \leq \sum_{l \sim 2^{i}} w(l)\left|\sum_{n \leq x / l} f(n) e(\alpha l n)\right|^{2} \\
& =\sum_{n, m \leq x / 2^{i}} f(n) \overline{f(m)} \sum_{\substack{l \sim 2^{i} \\
l \leq \min (x / n, x / m)}} w(l) e(\alpha(n-m) l) .
\end{aligned}
$$

To estimate the last sum in (2.8) we first observe that if $(b, q)=1$, then (2.7) and the Siegel-Walfisz theorem yield

$$
\sum_{\substack{l \leq t \\ l \equiv b(\bmod q)}} w(l)=\frac{t}{\varphi(q)}+O\left(\frac{t}{(\log t)^{10}}\right)
$$

while if $(b, q)=d>1$, say $b=d b^{\prime}$, then (2.7) gives

$$
\begin{aligned}
\sum_{\substack{l \leq t \\
l \equiv b(\bmod q)}} w(l) & =\sum_{\substack{k \leq t / d \\
k \equiv b^{\prime}(\bmod q / d)}} w(d k)=\frac{q}{\varphi(q)}\left(\frac{t / d}{q / d}+O(1)\right)+O(\log q) \\
& =\frac{t}{\varphi(q)}+O(\log q)
\end{aligned}
$$

and hence in either case we have

$$
\sum_{\substack{l \leq t \\ l \equiv b(\bmod q)}} w(l)=\frac{t}{\varphi(q)}+O\left(\frac{t}{(\log t)^{10}}\right),
$$


for $t \geq(\log q)^{2}$. Therefore, for $t \geq(\log q)^{2}$, we have

$$
\begin{aligned}
\sum_{l \leq t} w(l) e\left(\frac{a k}{q} l\right) & =\sum_{b=1}^{q} e\left(\frac{a k}{q} b\right)\left(\frac{t}{\varphi(q)}+O\left(\frac{t}{(\log t)^{10}}\right)\right) \\
& =\delta_{q}(k) \frac{q}{\varphi(q)} t+O\left(\frac{q t}{(\log t)^{10}}\right)
\end{aligned}
$$

with

$$
\delta_{q}(k)= \begin{cases}0 & \text { if } q \nmid k, \\ 1 & \text { if } q \mid k,\end{cases}
$$

from which it now readily follows by (2.2) and summation by parts (see, for example, [Va, Proof of Lemma 3.1]) that for $t>2^{i}$ we also have

$$
\begin{aligned}
& \sum_{\substack{l \sim 2^{i} \\
l \leq t}} w(l) e(\alpha k l) \\
& \ll \delta_{q}(k) \frac{q}{\varphi(q)} \min \left(2^{i}, \frac{1}{|\beta| \cdot|k|}\right)+\frac{q 2^{i}}{\left(\log 2^{i}\right)^{10}}+|\beta| \cdot|k| \frac{q 2^{2 i}}{\left(\log 2^{i}\right)^{10}} .
\end{aligned}
$$

The last estimate corresponds to our earlier estimate [Ba2, (2.5)].

An easy calculation shows that

$$
\frac{q 2^{i}}{\left(\log 2^{i}\right)^{10}}+|\beta| \cdot|k| \frac{q 2^{2 i}}{\left(\log 2^{i}\right)^{10}} \ll \frac{q}{\varphi(q)} \min \left(2^{i}, \frac{1}{|\beta| \cdot|k|}\right),
$$

for $x_{2} \leq 2^{i}<x_{3}$ and $|k| \leq x / 2^{i}$. We also observe that by the definition (2.3) of $\bar{x}$

$$
\min \left(2^{i}, \frac{1}{|\beta| \cdot|k|}\right) \ll \min \left(2^{i}, \frac{\bar{x}}{|k|}\right),
$$

for $|k| \leq x / 2^{i}$. Hence, by (2.5) and (2.8)-(2.12), we have, for $x_{2} \leq 2^{i}<x_{3}$,

$$
\sum_{p \sim 2^{i}} \log p\left|\sum_{n \leq x / p} f(n) e(\alpha p n)\right|^{2} \ll \sum_{1}+\sum_{2},
$$

where

$$
\sum_{1}=\sum_{\substack{n, m \leq x / 2^{i} \\ n \equiv m(\bmod q)}}|f(n)| \cdot|f(m)| \frac{q}{\varphi(q)} \min \left(2^{i}, \frac{\bar{x}}{|n-m|}\right)
$$

and

$$
\sum_{2}=\sum_{n, m \leq x / 2^{i}}|f(n)| \cdot|f(m)|\left(\frac{q 2^{i}}{\left(\log 2^{i}\right)^{10}}+|\beta| \cdot|n-m| \frac{q 2^{2 i}}{\left(\log 2^{i}\right)^{10}}\right) .
$$


Estimating $\sum_{2}$ trivially we obtain

$$
\begin{aligned}
\sum_{2} & \ll\left(\frac{x}{2^{i}}\right)^{2} \frac{q 2^{i}}{\left(\log 2^{i}\right)^{10}}+|\beta| \frac{q 2^{2 i}}{\left(\log 2^{i}\right)^{10}}\left(\frac{x}{2^{i}}\right)^{3} \\
& =(1+|\beta| x) \frac{q x^{2}}{2^{i}\left(\log 2^{i}\right)^{10}} \ll \frac{x}{\bar{x}} \cdot \frac{q x^{2}}{2^{i}\left(\log 2^{i}\right)^{10}},
\end{aligned}
$$

by $(2.3)$. For $\sum_{1}$ we now write

$$
\sum_{1} \ll \frac{q}{\varphi(q)} \sum_{1 \leq j \leq\lceil x / \bar{x}\rceil} \frac{2^{i}}{j} \sum_{\substack{n, m \leq x / 2^{i} \\(j-1) \bar{x} / 2^{i} \leq|n-m|<j \bar{x} / 2^{i} \\ n \equiv m(\bmod q)}}|f(n)| \cdot|f(m)| .
$$

Finally, combining (2.4), (2.6) and (2.13)-(2.15) yields the estimate

$$
\begin{aligned}
& F(x, \alpha) \ll \frac{x}{\mathcal{L}}+\frac{1}{\mathcal{L}} \sum_{x_{2} \leq 2^{i}<x_{3}}\left(2^{2 i} \frac{q}{\varphi(q)} \sum_{1 \leq j \leq\lceil x / \bar{x}\rceil} \frac{1}{j}\right. \\
& \left.\times \quad \sum_{n, m \leq x / 2^{i}}|f(n)| \cdot|f(m)|+\frac{q x^{3}}{\bar{x}\left(\log 2^{i}\right)^{10}}\right)^{1 / 2} . \\
& \begin{array}{c}
(j-1) \bar{x} / 2^{i} \leq|n-m|<j \bar{x} / 2^{i} \\
n \equiv m(\bmod q)
\end{array}
\end{aligned}
$$

From this the statement of the lemma follows since

$$
\sum_{x_{2} \leq 2^{i}<x_{3}} \sqrt{\frac{q x^{3}}{\bar{x}\left(\log 2^{i}\right)^{10}}} \ll x \frac{\sqrt{q x / \bar{x}}}{\left(\log x_{2}\right)^{4}} \ll x,
$$

by $(2.5)$.

Theorems 4 and 5 follow readily from Lemma 1. For Theorem 3 we will also require the following bound of $\mathrm{P}$. Shiu [Sh], where $\mathcal{G}$ denotes the class of multiplicative functions $g$ satisfying $0 \leq g \leq 1$.

Lemma 2. Let $\varepsilon, 0<\varepsilon \leq 1 / 2$, be fixed. Then the inequality

$$
\sum_{\substack{x-y<n \leq x \\ n \equiv a(\bmod q)}} g(n) \ll_{\varepsilon} \frac{y}{\varphi(q) \mathcal{L}} e^{S_{q}(x)}
$$

holds uniformly for all $g \in \mathcal{G}, x \geq 3, x^{\varepsilon} \leq y \leq x, 1 \leq q \leq y^{1-\varepsilon}$, and all integers a (not necessarily coprime with $q)$.

In addition to these lemmas, Theorems 1 and 2 will also require the following estimate.

LEMMA 3. Let an arbitrary natural number $q$ and a pair of real numbers $\tau \geq 3$ and $\varepsilon>0$ be given. Then for any two sequences of real numbers $x_{i}$ 
and $y_{i}, 1 \leq i \leq \kappa$, satisfying the conditions

$$
y_{1} \geq(q \tau)^{2}, \quad y_{i+1} \geq 2 y_{i}, \quad \text { and } \quad y_{i} \leq x_{i} \leq \tau y_{i},
$$

and an arbitrary sequence of integers $a_{i}$, we have

$$
\sum_{1 \leq i \leq \kappa} \frac{1}{y_{i}} \sum_{\substack{x_{i}-y_{i}<n \leq x_{i} \\ n \equiv a_{i}(\bmod q)}} g(n) \ll_{\varepsilon} \frac{1}{\varphi(q)}\left(e^{S_{q}\left(x_{1}\right)}\left(\frac{\log x_{\kappa}}{\log x_{1}}\right)^{\varepsilon}+e^{S_{q}\left(x_{\kappa}\right)}\right),
$$

uniformly for all $g \in \mathcal{G}$.

Proof. See [Ba3, Theorem 0].

3. Proof of Theorems 4 and 5. Assume, as we may, that $x \geq x_{0}$. Then Lemma 1 and the trivial estimate $|f(n)| \leq 1$ immediately yield

$$
\begin{aligned}
F(x, \alpha) & \ll \frac{x}{\mathcal{L}}+\frac{1}{\mathcal{L}} \sqrt{\frac{q}{\varphi(q)}} \sum_{1 \leq 2^{i}<x /(q x / \bar{x})^{2}} 2^{i}\left(\frac{x \bar{x}}{q 2^{2 i}} \log \left(2 \frac{x}{\bar{x}}\right)\right)^{1 / 2} \\
& \ll \frac{x}{\mathcal{L}}+\frac{x}{\sqrt{\varphi(q)}} \sqrt{\frac{\log (2 x / \bar{x})}{x / \bar{x}}},
\end{aligned}
$$

and Theorem 4 follows.

To prove Theorem 5 let $a$ and $q$ be as in the statement of Theorem 4, so that (3.1) holds. From this and the well known bound $\varphi(q) \gg q / \log _{2} q$ the desired estimate follows immediately if $q \geq R / 2$. For $q<R / 2$ we have

$$
\frac{1}{q r} \leq\left|\frac{a}{q}-\frac{s}{r}\right|=\left|\frac{a}{q}-\alpha+\alpha-\frac{s}{r}\right| \leq|\beta|+\frac{1}{2 q r},
$$

by the definition (2.2) of $\beta$ and our hypotheses. This and the definition (2.3) of $\bar{x}$ give

$$
\frac{x}{\bar{x}} \geq|\beta| x \geq \frac{x}{2 q r} .
$$

This in turn yields the estimate

$$
\frac{1}{\varphi(q)} \cdot \frac{\log (2 x / \bar{x})}{x / \bar{x}} \ll \frac{1}{q / \log _{2} q} \cdot \frac{\log (x / r)}{x /(q r)} \ll \frac{\log R \log _{2} R}{R} .
$$

Applying (3.2) to the right-hand side of (3.1) gives the desired bound for $q<R / 2$, and thus completes the proof of the theorem.

4. Proof of Theorem 3. This theorem improves on the bound established by the author in [Ba2]. The improvement stems from the superior version of Lemma 1 given in this paper, with the rest of the proof differing from the argument in [Ba2] in straightforward details only. We, however, complete the argument here for the convenience of the reader. 
Again we assume that $x \geq x_{0}$ and appeal to Lemma 1. We have

$$
\begin{aligned}
F(x, \alpha) & \ll \frac{x}{\mathcal{L}}+\frac{1}{\mathcal{L}} \sqrt{\frac{q}{\varphi(q)}} \sum_{1 \leq 2^{i}<x_{3}} 2^{i} \\
& \times\left(\sum_{1 \leq j \leq\lceil x / \bar{x}\rceil} \frac{1}{j} \sum_{m \leq x / 2^{i}}|f(m)| \sum_{\substack{(j-1) \bar{x} / 2^{i} \leq n-m<j \bar{x} / 2^{i} \\
n \equiv m(\bmod q)}}|f(n)|\right)^{1 / 2},
\end{aligned}
$$

with $x_{3}$ given by (2.5). To estimate the innermost sum above we appeal to Lemma 2. One easily verifies that for $i$ in our range all of the hypotheses of this lemma are satisfied and we obtain, for every $m$, the bound

$$
\sum_{\substack{(j-1) \bar{x} / 2^{i} \leq n-m<j \bar{x} / 2^{i} \\ n \equiv m(\bmod q)}}|f(n)| \ll \frac{\bar{x} / 2^{i}}{\varphi(q) \log \left(2 \bar{x} / 2^{i}\right)} e^{S(x)},
$$

since $|f| \leq 1$. With this we estimate the summation over $i$ in (4.1) by

$$
\ll\left(\frac{\bar{x} \log (2 x / \bar{x})}{\varphi(q)} e^{S(x)}\right)^{1 / 2} \sum_{1 \leq 2^{i}<x_{3}}\left(\frac{2^{i}}{\log \left(2 \bar{x} / 2^{i}\right)} \sum_{m \leq x / 2^{i}}|f(m)|\right)^{1 / 2} .
$$

Applying Cauchy's inequality we get (see [Ba2, (3.2)])

$$
\sum_{1 \leq 2^{i}<x_{3}}\left(\frac{2^{i}}{\log \left(2 \bar{x} / 2^{i}\right)} \sum_{m \leq x / 2^{i}}|f(m)|\right)^{1 / 2} \ll\left(x e^{S(x)} \mathcal{L}_{2}\right)^{1 / 2} .
$$

Thus, by (4.1)-(4.3), we obtain

$$
F(x, \alpha) \ll \frac{x}{\mathcal{L}}+\frac{x}{\sqrt{q} \mathcal{L}} e^{S(x)} \frac{q}{\varphi(q)}\left(\frac{\log (2 x / \bar{x})}{x / \bar{x}} \mathcal{L}_{2}\right)^{1 / 2} .
$$

The theorem now follows by the same argument that was used to derive Theorem 5 from (3.1) in the previous section.

5. Proof of Theorems 1 and 2. Here we further refine arguments of the last section this time by combining Lemma 1 with Lemma 3. To this end we assume, as before, that $x \geq x_{0}$ and let the quantities $q, \bar{x}$ and $x_{3}$ be defined by (2.1), (2.3) and (2.5), respectively. We will now show that the estimate

$$
F(x, \alpha) \ll_{\varepsilon} \frac{x}{\mathcal{L}}+\frac{x}{\sqrt{q} \mathcal{L}}\left(\mathcal{L}^{\varepsilon}+e^{S_{q}(x)}\right)\left(\frac{q}{\varphi(q)}\right)^{3 / 2} \sqrt{\frac{\log (2 x / \bar{x})}{x / \bar{x}}}
$$

holds. From this estimate Theorem 1 follows immediately, in view of the inequality $\varphi(q) \gg q / \log _{2} q$, while Theorem 2 is deduced by an argument 
analogous to the one we used to deduce Theorem 5 from (3.1) in Section 3. Thus it only remains to prove (5.1).

Appealing to (0.4), as in the proof of Lemma 1, allows us to consider only the case when $q<\mathcal{L}^{3}$, as we now assume. Now, for $i$ in the range $1 \leq 2^{i}<x_{3}$, define integer sequences $l_{i}$ and $b_{i}$ by

$$
\sum_{\substack{\left(l_{i}-2\right) \bar{x} / 2^{i}<n \leq l_{i} \bar{x} / 2^{i} \\ n \equiv b_{i}(\bmod q)}}|f(n)|=\max _{\substack{1 \leq l \leq 2\lceil x / \bar{x}\rceil \\ 1 \leq b \leq q}} \sum_{\substack{(l-2) \bar{x} / 2^{i}<n \leq l \bar{x} / 2^{i} \\ n \equiv b(\bmod q)}}|f(n)| .
$$

With these definitions we write

$$
\begin{aligned}
& \sum_{1 \leq j \leq\lceil x / \bar{x}\rceil} \frac{1}{j} \sum_{\substack{n, m \leq x / 2^{i} \\
(j-1) \bar{x} / 2^{i} \leq|n-m|<j \bar{x} / 2^{i} \\
n \equiv m(\bmod q)}}|f(n)| \cdot|f(m)| \\
& \ll \sum_{1 \leq j, k \leq\lceil x / \bar{x}\rceil} \frac{1}{j} \sum_{1 \leq b \leq q} \sum_{\begin{array}{c}
(k-1) \bar{x} / 2^{i}<m \leq k \bar{x} / 2^{i} \\
m \equiv b(\bmod q)
\end{array}}|f(m)| \\
& \times \sum_{\substack{(k+j-2) \bar{x} / 2^{i}<n \leq(k+j) \bar{x} / 2^{i} \\
n \equiv b(\bmod q)}}|f(n)| \\
& \ll q \frac{x}{\bar{x}} \log \left(2 \frac{x}{\bar{x}}\right)\left(\sum_{\substack{\left(l_{i}-2\right) \bar{x} / 2^{i}<n \leq l_{i} \bar{x} / 2^{i} \\
n \equiv b_{i}(\bmod q)}}|f(n)|\right)^{2},
\end{aligned}
$$

for $1 \leq 2^{i}<x_{3}$. Thus, by Lemma 1 , we have

$$
\begin{aligned}
& F(x, \alpha) \ll \frac{x}{\mathcal{L}}+\frac{x}{\sqrt{q} \mathcal{L}} \sqrt{\frac{\log (2 x / \bar{x})}{x / \bar{x}}} \cdot \frac{q^{3 / 2}}{\sqrt{\varphi(q)}} \\
& \times \sum_{1 \leq 2^{i}<x_{3}} \frac{1}{\bar{x} / 2^{i}} \sum_{\substack{\left(l_{i}-2\right) \bar{x} / 2^{i}<n \leq l_{i} \bar{x} / 2^{i} \\
n \equiv b_{i}(\bmod q)}}|f(n)| .
\end{aligned}
$$

To prepare for the application of Lemma 3 we set

$$
\tau=4 \frac{x}{\bar{x}}
$$

so that, by (2.5),

$$
x_{3}=\frac{x}{(q x / \bar{x})^{2}}=16 \frac{x}{q^{2} \tau^{2}},
$$

and write 


$$
\begin{array}{r}
\left(\sum_{1 \leq 2^{i} \leq x_{3} /(4 \tau)}+\sum_{x_{3} /(4 \tau)<2^{i}<x_{3}}\right) \frac{1}{\bar{x} / 2^{i}} \sum_{\begin{array}{c}
\left(l_{i}-2\right) \bar{x} / 2^{i}<n \leq l_{i} / 2^{i} \\
n \equiv b_{i}(\bmod q)
\end{array}}|f(n)| \\
=\sum_{1}+\sum_{2},
\end{array}
$$

say. We first dispose of $\sum_{2}$ whose inner sum we estimate by means of Lemma 2. Recalling that the applicability of this lemma was already verified in the proof of Theorem 3 we obtain, by (5.3) and (5.4),

$$
\sum_{2} \ll \frac{1}{\varphi(q)} e^{S_{q}(x)} \sum_{x_{3} /(4 \tau)<2^{i}<x_{3}} \frac{1}{\log \left(2 \bar{x} / 2^{i}\right)} \ll \frac{1}{\varphi(q)} e^{S_{q}(x)} .
$$

Next we estimate $\sum_{1}$ by Lemma 3 . The shortening of the range of the original summation to $1 \leq 2^{i} \leq x_{3} /(4 \tau)$ was designed to guarantee that $\sum_{1}$ satisfies all the hypotheses of that lemma with $\tau$ given by (5.3), as one readily verifies. Furthermore, a simple calculation shows that, in the language of Lemma 3 , we have $x_{\kappa} \leq 4 x$ and $x_{1} \leq 2 \tau^{3} q^{2}$. Noting that $S_{q}(4 x)=S_{q}(x)+O(1)$ and that, by Mertens's estimate, the hypothesis $q<\mathcal{L}^{3},(5.3)$ and $(2.1)-(2.3)$,

$$
e^{S_{q}\left(x_{1}\right)} \ll \log x_{1} \ll \log \tau+\log q \ll \mathcal{L}_{2},
$$

we thus obtain the bound

$$
\sum_{1} \ll_{\varepsilon} \frac{1}{\varphi(q)}\left(\mathcal{L}^{\varepsilon}+e^{S_{q}(x)}\right) .
$$

Finally, combining (5.2) and (5.5)-(5.7) yields (5.1) and hence completes the proof of Theorems 1 and 2 .

6. Examples. In this section we construct functions $f \in \mathcal{F}$ which verify the validity of propositions (i)' and (ii)' of Section 1 . As we have already mentioned, our examples are very simple modifications of examples constructed by Montgomery and Vaughan to establish the corresponding propositions (i) and (ii). Moreover, an argument used in [MV] to deduce (iii) from (ii) can now be used, with obvious modifications, to deduce (iii) ${ }^{\prime}$ from (ii) ${ }^{\prime}$, and thus will be omitted.

Recall that a real number $\lambda, 0<\lambda \leq 1$, is fixed. Given $x \geq 3$ set

$$
y=e^{\mathcal{L}^{1-\lambda}} \text {. }
$$

Let $\mathbf{1}_{y}$ be the characteristic function of the natural numbers all of whose prime factors are greater than $y$, i.e. $\mathbf{1}_{y}$ is completely multiplicative with

$$
\mathbf{1}_{y}= \begin{cases}0 & \text { if } p \leq y \\ 1 & \text { if } p>y\end{cases}
$$

We will need several facts about the function $\mathbf{1}_{y}$ which we now establish. 
In the first place we have, by Mertens's estimate and (6.1),

$$
\sum_{p \leq x} \frac{\mathbf{1}_{y}(p)}{p}=\lambda \mathcal{L}_{2}+O(1) .
$$

Furthermore, a "fundamental lemma" from sieve theory (see for example [HR, Theorem 2.5]) gives

$$
\begin{aligned}
& \sum_{\substack{n \leq x \\
n \equiv b(\bmod q)}} \mathbf{1}_{y}(n) \\
& \quad=\frac{x}{q} W_{q}(y)\left\{1+O\left(\exp \left(-\frac{\log (x / q)}{\log y}\right)+\exp (-\sqrt{\log (x / q)})\right)\right\},
\end{aligned}
$$

for any coprime natural numbers $b$ and $q$, where

$$
W_{q}(y)=\prod_{\substack{p \leq y \\(p, q)=1}}\left(1-\frac{1}{p}\right) .
$$

Thus, for $q \leq \sqrt{x}$, we obtain, by (6.1), the estimates

$$
\sum_{\substack{n \leq x \\ n \equiv b(\bmod q)}} \mathbf{1}_{y}(n)=\frac{x}{q} W_{q}(y)\left(1+O\left(e^{-\mathcal{L}^{\lambda / 3}}\right)\right)
$$

and

$$
\sum_{\substack{n \leq x \\(n, q)=1}} \mathbf{1}_{y}(n)=\varphi(q) \frac{x}{q} W_{q}(y)\left(1+O\left(e^{-\mathcal{L}^{\lambda / 3}}\right)\right) .
$$

We now use these estimates to modify the examples of Montgomery and Vaughan as follows.

(i)' Consider the sum

$$
\sum_{n \leq x} \mathbf{1}_{y}(n) z^{\Omega(n)} e(\alpha n)+\sum_{x / 2<p \leq x}(1-z e(\alpha p)),
$$

where $\Omega(n)$ denotes the total number of prime factors of $n$. From the estimate

$$
\sum_{x / 2<p \leq x} 1 \gg x / \mathcal{L}
$$

and the maximum modulus principle it follows that for some $z_{0},\left|z_{0}\right|=1$, the modulus of the expression in $(6.6)$ is $\gg x / \mathcal{L}$. From this and $(6.2)$ we deduce that the completely multiplicative function $f$ given by

$$
f(p)= \begin{cases}\mathbf{1}_{y}(p) z_{0} & \text { if } p \leq x / 2, \\ e(-\alpha p) & \text { if } p>x / 2\end{cases}
$$


satisfies

$$
|F(x, \alpha)| \gg x / \mathcal{L}
$$

and

$$
S(x)=\sum_{p \leq x} \frac{|f(p)|}{p}=\lambda \mathcal{L}_{2}+O(1) .
$$

(ii)' Let $\chi$ be a character modulo $q$ induced by the primitive character $\chi^{*}$ modulo $k$, so that $k \mid q$. Set $f(n)=\mathbf{1}_{y}(n) \chi(n)$. Now write

$$
F\left(x, \frac{a}{q}\right)=\sum_{1 \leq b \leq q} \chi(b) e\left(\frac{a}{q} b\right) \sum_{\substack{n \leq x \\ n \equiv b(\bmod q)}} \mathbf{1}_{y}(n) .
$$

For $(b, q)=1$ we estimate the last summation over $n$ by (6.4) to obtain

$$
\begin{aligned}
F\left(x, \frac{a}{q}\right) & =\frac{x}{q} W_{q}(y)\left(\sum_{1 \leq b \leq q} \chi(b) e\left(\frac{a}{q} b\right)+O\left(q e^{-\mathcal{L}^{\lambda / 3}}\right)\right) \\
& =\frac{x}{q} W_{q}(y)\left(\overline{\chi(a)} \tau(\chi)+O\left(q e^{-\mathcal{L}^{\lambda / 3}}\right)\right),
\end{aligned}
$$

where $\tau(\chi)$ denotes Gauss' sum. Since there are no primitive characters modulo $q$ if and only if $q \equiv 2(\bmod 4)$ (see, for example, [Da, §4]) we choose $\chi$ so that $k=q / 2$ in this case and $k=q$ otherwise. In either case (6.7) together with the well known identities

$$
\tau(\chi)=\mu(q / k) \chi^{*}(q / k) \tau\left(\chi^{*}\right) \text { and }\left|\tau\left(\chi^{*}\right)\right|=\sqrt{k}
$$

(see, for example, [Da, $\S 9]$ ) now yield, for $q \leq e^{\mathcal{L}^{\lambda / 3}}$,

$$
F\left(x, \frac{a}{q}\right) \gg \frac{x}{\sqrt{q}} W_{q}(y)
$$

provided that $x$ is sufficiently large in terms of $\lambda$. Combining this with (6.5) and (6.3) we obtain, for $x$ sufficiently large in terms of $\lambda$,

$$
\begin{aligned}
F\left(x, \frac{a}{q}\right) & \gg \frac{1}{\sqrt{q}} \sum_{\substack{n \leq x \\
(n, q)=1}}|f(n)| \frac{q}{\varphi(q)} \\
& \gg \frac{x}{\sqrt{q}} W_{q}(y) \\
& =\frac{x}{\sqrt{q}} \prod_{p \leq x}\left(1-\frac{1}{p}\right) \prod_{\substack{y<p \leq x \\
(p, q)=1}}\left(1-\frac{1}{p}\right)^{-1} \prod_{p \mid q}\left(1-\frac{1}{p}\right)^{-1} \\
& \gg \frac{x}{\sqrt{q} \mathcal{L}} e^{S_{q}(x)} \frac{q}{\varphi(q)},
\end{aligned}
$$

as desired. 


\section{References}

[Ba1] G. Bachman, Exponential sums with multiplicative coefficients, Electron. Res. Announc. Amer. Math. Soc. 5 (1999), 128-135.

[Ba2] -, On exponential sums with multiplicative coefficients in: Number Theory (Halifax, NS, 1994), CMS Conf. Proc. 15, Amer. Math. Soc., Providence, RI 1995, $29-38$.

[Ba3] -, On a Brun-Titchmarsh inequality for multiplicative functions, this issue, $1-25$.

[Da1] H. Daboussi, Fonctions multiplicatives presque périodiques B. D'après un travail commun avec Hubert Delange, Astérisque 24-25 (1975), 321-324.

[Da2] -, On some exponential sums, in: Analytic Number Theory (Allerton Park, IL, 1989), Progr. Math. 85, B. C. Berndt, H. G. Diamond, H. Halberstam and A. Hildebrand (eds.), Birkhäuser, Boston, MA, 1990, 111-118.

[DD1] H. Daboussi et H. Delange, Quelques propriétés des fonctions multiplicatives de module au plus égal à 1, C. R. Acad. Sci. Paris Sér. A 278 (1974), 657-660.

[DD2] - - - On multiplicative arithmetical functions whose modulus does not exceed one, J. London Math. Soc. (2) 26 (1982), 245-264.

[Da] H. Davenport, Multiplicative Number Theory, 2nd ed., Springer, New York, 1980.

[DHT] Y. Dupain, R. R. Hall et G. Tenenbaum, Sur l'équirépartition modulo 1 de certaines fonctions de diviseurs, J. London Math. Soc. (2) 26 (1982), 397-411.

[FT] E. Fouvry et G. Tenenbaum, Entiers sans grand facteur premier en progressions arithmétiques, Proc. London Math. Soc. 63 (1991), 449-494.

[Go] L. Goubin, Sommes d'exponentielles et principe de l'hyperbole, Acta Arith. 73 (1995), 303-324.

[HR] H. Halberstam and H.-E. Richert, Sieve Methods, Academic Press, London, 1974.

[Ha] R. R. Hall, Halving an estimate obtained from Selberg's upper bound method, Acta Arith. 25 (1974), 347-351.

[MV] H. L. Montgomery and R. C. Vaughan, Exponential sums with multiplicative coefficients, Invent. Math. 43 (1977), 69-82.

[Sh] P. Shiu, A Brun-Titchmarsh theorem for multiplicative functions, J. Reine Angew. Math. 313 (1980), 161-170.

[Te] G. Tenenbaum, Facteurs premiers de sommes d'entiers, Proc. Amer. Math. Soc. 106 (1989), 287-296.

[Va] R. C. Vaughan, The Hardy-Littlewood Method, 2nd ed., Cambridge Tracts in Math. 125, Cambridge Univ. Press, Cambridge, 1997.

Department of Mathematical Sciences

University of Nevada, Las Vegas

4505 Maryland Parkway

Las Vegas, NV 89154-4020, U.S.A.

E-mail: bachman@unlv.edu 